\title{
Helmintos, myxozoos y microsporidios en músculos de peces comercializados frescos y su importancia como riesgo potencial para la salud humana en la ciudad de Valdivia, Chile ${ }^{\#}$
}

\author{
Helminths, myxozoans and microsporidians in muscles of commercialised fresh fish and their \\ importance as potential risk for human health in the city of Valdivia, Chile
}

\author{
P Torres ${ }^{\mathrm{a}}$, S Puga ${ }^{\mathrm{a}}$, L Castillo ${ }^{\mathrm{a}}, \mathrm{J}$ Lamilla ${ }^{\mathrm{b}}, \mathrm{JC}_{\text {Miranda }}^{\mathrm{c}}$ \\ anstituto de Parasitología, Facultad de Medicina, Universidad Austral de Chile, Valdivia, Chile. \\ ${ }^{b}$ Instituto de Ciencias Marinas y Limnológicas, Facultad de Ciencias, Universidad Austral de Chile, Valdivia, Chile. \\ 'Instituto de Estadística, Facultad de Ciencias Económicas y Administrativas, Universidad Austral de Chile, Valdivia, Chile.
}

\section{SUMMARY}

The prevalence, mean intensity, mean abundance, and mean density of infection by helminths, myxozoans, and microsporidians were determined in the muscles of southern hake, Merluccius australis $(\mathrm{n}=104)$, pink cusk-eel, Genypterus blacodes $(\mathrm{n}=81)$, and snoek, Thyrsites atun $(\mathrm{n}=95)$, all of them sold fresh in the city of Valdivia, Chile. Fish were examined on a candling table, with direct observation of the dorsal and ventral muscles (candling 1), in cuts $4 \mathrm{~mm}$ thick or less (candling 2), and on a glass plate compression. Prevalence, mean intensity, and mean abundance of infection, by all the identified parasites in each host, were significantly higher $(\mathrm{P}<0.05)$ in hake and pink cusk-eel, compared with the snoek. Mean density of infection in the hake was higher $(\mathrm{P}<0.05)$ than in pink cusk-eel; the latter had a higher $(\mathrm{P}<0.05)$ density of infection than the snoek. All fish hosts showed parasites with potential risks to human health, such as Anisakis sp. types I and II or Pseudoterranova sp. Other parasites were also detected in each fish host: metacercariae of trematodes, Hepatoxylon trichiuri, Pseudoterranova sp., Anisakis sp. type I, Kudoa sp., and microsporidians in the hake; metacercariae of trematodes, Pseudoterranova sp., Anisakis sp. types I and type II, Corynosoma sp., Kudoa sp., and microsporidians in pink cusk-eel; and Molicola sp., Pseudoterranova sp., and Anisakis sp. type I in the snoek. Pseudoterranova sp. and Molicola sp. had the highest prevalence, mean intensity, mean abundance, and mean density of infection in the pink cush-eel and the snoek, respectively. In the hake, the highest prevalence, mean abundance and mean density were determined for Kudoa sp; mean intensity of infection was highest for microsporidiosis.

Key words: parasites, zoonoses, fish muscles, food.

\section{RESUMEN}

La prevalencia e intensidad, abundancia y densidad media de infección por helmintos, myxozoos y microsporidios fue determinada en músculos de merluza austral, Merluccius australis $(\mathrm{n}=104)$, congrio dorado, Genypterus blacodes $(\mathrm{n}=81)$ y sierra, Thyrsites atun $(\mathrm{n}=95)$, comercializados frescos en la ciudad de Valdivia, Chile. El análisis fue realizado en mesa de candling, observando directamente la musculatura dorsal y ventral (candling 1), en cortes de $4 \mathrm{~mm}$ o menos de espesor (candling 2) y por compresión en placas. La prevalencia, intensidad media y abundancia media de infección, por el total de parásitos determinados en cada huésped, resultaron significativamente mayores $(\mathrm{P}<0,05)$ en la merluza y el congrio, respecto a la sierra. La densidad media fue significativamente mayor $(\mathrm{P}<0,05)$ en la merluza respecto al congrio y en este último resultó mayor $(\mathrm{P}<0,05)$ que en la sierra. Las tres especies de peces presentaron parásitos de riesgo potencial para la salud humana, como Anisakis sp. tipos I y II o Pseudoterranova sp. Los siguientes parásitos fueron determinados en cada huésped: metacercarias de trematodos, Hepatoxylon trichiuri, Pseudoterranova sp., Anisakis sp. tipo I, Kudoa sp. y microsporidios no identificados en merluza; metacercarias, Pseudoterranova sp., Anisakis sp. tipos I y II, Corynosoma sp., Kudoa sp. y microsporidios no identificados en el congrio; y Pseudoterranova sp., Anisakis sp. tipo I y Molicola sp. en la sierra. La mayor prevalencia e intensidad, abundancia y densidad media de infección fue para Pseudoterranova sp. y Molicola sp. en congrio y sierra, respectivamente. La mayor prevalencia, abundancia y densidad media de infección en merluzas correspondió a Kudoa sp., y la mayor intensidad de infección fue observada para la microsporidiosis.

Palabras clave: parásitos, zoonosis, músculos de peces, alimento.

\section{INTRODUCCIÓN}

En los últimos años el consumo de pescado de origen marino se ha incrementado en muchos países, en parte,

Aceptado: 20.06.2013.

\# Financiado parcialmente por el programa sobre Diversidad de parásitos y zoonosis transmitidas por organismos acuáticos, y DIDUACH (I-2010-02).

* Casilla 567, Valdivia, Chile; ptorres@uach.cl. por los regímenes de dieta saludable para prevenir enfermedades cardiovasculares y cáncer (Broglia y Kapel 2011), favoreciendo una mayor exposición a infecciones por helmintos parásitos (trematodos, cestodos, nematodos y acantocéfalos). Alrededor de un tercio de las especies de helmintos identificadas en el hombre (Coombs y Crompton 1991) pueden ser transmitidas por el consumo de pescado crudo (cebiche, sushi, sashimi y otros similares), ahumado en frío, salado, escabechado (marinado) o 
insuficientemente cocido (Torres 2012).

La anisakidosis causada por nematodos de algunos géneros (Anisakis, Pseudoterranova, Contracaecum e Hysterothylacium) de la familia Anisakidae, es una de las mas severas y provoca enfermedad gástrica e intestinal y reacciones alérgicas (Ceballos-Mandiola y col 2010), considerándose actualmente un riesgo emergente para la salud y seguridad alimentaria (Kleter y col 2009). La anisakidosis humana causó alrededor de 31.575 casos en el mundo hasta 1996 (Takahashi y col 1998), registrándose en Japón, Corea, Taiwán, Israel, Egipto, Omán, Tailandia, Holanda, Francia, España, Alemania, Bélgica, Noruega, Polonia, Reino Unido, Suecia, Dinamarca, Finlandia, Groenlandia, Canadá, Estados Unidos, Méjico, Puerto Rico, Venezuela, Perú, Chile, Australia, Nueva Zelandia, Samoa y Tahití (Takahashi y col 1998, Audicana y col 2003, Jimenez-Huyke y col 2005, Shamsi y Butcher 2011, Torres 2012). La mayoría de los casos de anisakidosis son causados por especies de Anisakis y Pseudoterranova (Adams y col 1997, Takahashi y col 1998, Audicana y col 2003). Los productos de excreción y secreción de Anisakis simplex (sensu lato) pueden ser resistentes al congelamiento y cocción, causando cuadros alérgicos a consumidores, cocineros o procesadores de pescado (Audicana y col 2003, Ventura y col 2008, Lin y col 2012).

En Chile, entre las infecciones transmitidas por peces marinos, la anisakidosis es considerada emergente y es provocada por larvas de Pseudoterranova sp. y Anisakis $\mathrm{sp}$., publicándose alrededor de 30 casos humanos (Torres y col 2007, Jofré y col 2008); dichas larvas han sido identificadas en 21 y 37 especies de peces, respectivamente (Muñoz y Olmos 2008). La diphyllobothriosis por el cestodo Diphyllobothrium pacificum, con ciclo de vida marino, se menciona en 16 casos humanos en Chile (Sagua y col 2001); identificándose plerocercoides de Diphyllobothrium sp. en truchas cultivadas, Oncorhynchus mykiss, y en salmones silvestres de retorno, Oncorhynchus kisutch (Torres y col 2010). En corvinas, Cilus gilberti, congrio dorado, Genypterus maculatus y merluza austral Merluccius australis, se han registrado plerocercoides del orden Diphyllobothridea, sin determinar su género (Muñoz y Olmos 2008).

Otros parásitos de peces marinos de la costa de Chile (Muñoz y Olmos 2008), también han sido asociados a riesgos para la salud humana en el hemisferio norte. Productos somáticos, de excreción o secreción de larvas de cestodos del orden Trypanorhyncha han demostrado tener efecto tóxico (Deardorff y col 1984) y alergénico (Gómez-Morales y col 2008, Pelayo y col 2009). En pacientes españoles, consumidores de pescado, con trastornos gastrointestinales y alérgicos se han observado pruebas cutáneas positivas a extractos antigénicos de Kudoa sp. (Myxozoa) (Martinez de Velasco y col 2008); también, el consumo de lenguados crudos (Paralichthys olivaceus), infectados con Kudoa septempunctata, ha sido responsable de brotes de gastroenteritis en Japón (Kawai y col 2012).
Las larvas de anisákidos se localizan en los músculos, vísceras y cavidad corporal de los peces pudiendo desplazarse, desde las dos últimas localizaciones, a los músculos después de muertos (Smith y Wootten 1975). Para descartar la presencia de larvas de anisákidos, la legislación de distintos países, como aquellos de la Comunidad Europea (Llarena-Reino y col 2012), Estados Unidos (FDA 2009), o Chile (SERNAPESCA, 2012) recomiendan la inspección visual de los peces en los centros de procesamiento. En Chile, los peces frescos refrigerados de exportación se someten a un examen mediante la técnica de candling para verificar la presencia de parásitos causantes de zoonosis, como larvas de anisákidos y plerocercoides de Diphyllobothrium spp. (SERNAPESCA 2012). Por otra parte, el reglamento sanitario de los alimentos (RSA 2011) señala que el pescado que se comercialice para el consumo humano en el país, además de estar refrigerado, debe estar exento de parásitos y sus quistes. En Chile, en peces comercializados frescos, como merluzas, Merluccius gayi y Macrouronus magellanicus, congrio colorado (Genypterus chilensis), lenguado (Paralichthys microps) y jurel, (Trachurus murphyi) se efectuó un estudio cuantitativo de anisákidos en sus músculos (Torres y col 2000), mediante la técnica de compresión en placas. Más tarde, dicha técnica demostró una eficacia significativamente mayor respecto a la técnica de candling en la búsqueda de Diphyllobothrium spp. en $O$. mykiss (Torres y Puga 2011).

Merluccius australis, G. blacodes y la sierra, Thyrsites atun se encuentran entre las especies de peces de importancia económica y de consumo frecuente en Chile de las cuales se extraen alrededor de 25.000, 4.000 y $2.556 \mathrm{t}$ anuales, respectivamente (Arana 2012). Las dos primeras especies se exportan frescas refrigeradas o congeladas, principalmente a España y Portugal, mientras que T. atun solo es de consumo interno. Si bien se mencionan estudios parasitológicos generales en tales especies (Muñoz y Olmos 2008), no se registran trabajos a nivel del producto comercializado fresco y refrigerado en los mercados nacionales, mediante aplicación de tecnologías de alta sensibilidad para detectar y cuantificar parásitos eucarióticos en el tejido muscular.

Los objetivos de este estudio son: 1) Determinar los helmintos, myxozoos y microsporidios en músculos de la merluza austral, congrio dorado y sierra comercializados frescos en la ciudad de Valdivia $\left(30^{\circ} 48^{\prime} 30^{\prime \prime} \mathrm{S}\right.$, $\left.73^{\circ} 14^{\prime} 30^{\prime \prime} \mathrm{O}\right)$ Chile, así como identificar aquellos de riesgo potencial para la salud humana y 2) comparar la prevalencia e intensidad, abundancia y densidad media de infección por los taxones determinados en cada especie de pez.

\section{MATERIAL Y MÉTODOS}

Entre julio de 2008 y enero de 2009 se examinaron los músculos de 280 peces eviscerados y comercializados frescos en los mercados de la ciudad de Valdivia. En el 
cuadro 1 se indican las especies, número de ejemplares examinados, longitud estándar y peso corporal.

De cada pez, se separó la musculatura ventral y dorsal para examinar, cada una, en una mesa de candling con cubierta de vidrio de $30 \times 60 \mathrm{~cm}$ y equipada con tres ampolletas de luz blanca de $25 \mathrm{~W}$, situadas a $20 \mathrm{~cm}$ debajo de la superficie de vidrio (candling 1), aislando cada parásito. Posteriormente, en cada porción muscular se hicieron cortes de $4 \mathrm{~mm}$ o menos, examinándose en la mesa de candling (candling 2), y aislando los parásitos observados. Los cortes de cada región fueron finalmente observados mediante compresión manual entre dos placas de vidrio de $18 \times 10 \times 0,8 \mathrm{~cm}$, usando un microscopio estereoscópico y aislando los parásitos determinados (Torres y Puga 2011).

Las larvas de nematodos fueron fijadas en formaldehido de $4 \%$ en $\mathrm{Na} \mathrm{Cl}$ de $1 \%$ (formol-salino) y diafanizadas en lactofenol para su estudio morfológico mediante microscopio de luz, de acuerdo a Berland (1961) y Petter y Maillard (1988). Los cistacantos de acantocéfalos fueron depositados en agua a $4{ }^{\circ} \mathrm{C}$ por $12 \mathrm{~h}$, fijados y diafanizados de forma similar a los nematodos. Las larvas de cestodos fueron observadas en preparaciones frescas en microscopio estereoscópico y luego fijadas como los nematodos. Los plerocercoides de Trypanorhyncha fueron analizados directamente en microscopio estereoscópico. Los merocercoides, puestos en lactofenol, fueron disecados, observándose detalles de sus tentáculos, botridios y otras estructuras del escólex en microscopio de luz. Para la identificación de las larvas de Trypanorhyncha se siguió a Robinson (1959) y Palm (2004). Con las metacercarias y los quistes de Myxozoa y Microsporidia se realizaron preparaciones microscópicas frescas con $\mathrm{NaCl} 0,15 \mathrm{M}$ y observadas con microscopio de luz.

La aplicación de los descriptores de prevalencia, intensidad media, abundancia media y densidad media de infección siguieron las recomendaciones de Bush y col (1997). Prevalencia corresponde al número de peces infectados con uno o más individuos de una especie particular o grupo taxonómico dividido por el número de huéspedes examinados, expresada como porcentaje. Intensidad media es la media de parásitos de una especie o grupo taxonómico identificado en una especie de huésped dividido por el número de individuos infectados. Abundancia media, a diferencia de la intensidad media, considera el total de individuos examinados de una especie de huésped. Densidad corresponde al número de parásitos de un taxón particular por cada $100 \mathrm{~g}$ de músculo en un individuo huésped. Densidad media es el promedio de densidades en el total de individuos examinados de una especie huésped.

Para los análisis de prevalencia de infección, por el total de taxones parasitarios de cada especie de pez, se aplicó la prueba de $\chi^{2}$ de Pearson para dos o tres muestras. El análisis de varianza mediante la prueba de Kruskall-Wallis fue usada para tres muestras y la prueba $\mathrm{U}$ de Mann Whitney, corregida por Bonferroni, se aplicó a pares de muestras (Sachs 1968), en las comparaciones de intensidad, abundancia y densidad media de infección. Para todas las pruebas, $\mathrm{P}<0,05$ fue considerada estadísticamente significativa. Para los análisis de prevalencias se utilizó el programa EPI DAT 3.1 ${ }^{\mathrm{TM}}$ Dirección Xeral de Saúde Pública de la Consellería de Sanidade, Xunta de Galicia, España/OPS-OMS.

\section{RESULTADOS}

La prevalencia de infección, al considerar el total de taxones identificados en cada huésped, mostró diferencias significativas $(\mathrm{P}<0,05)$ (cuadro 2$)$. Las prevalencias de infección en $M$. australis y G. blacodes fueron similares $(\mathrm{P}>0,05)$, pero, significativamente mayores ( $\mathrm{P}$ $<0,05$ ) respecto a $T$. atun (cuadro 2). La intensidad, abundancia y densidad media de infección, por el total de taxones identificados en cada huésped, también mostraron diferencias significativas $(\mathrm{P}<0,05)$; resultando la intensidad y abundancia media similar $(\mathrm{P}>0,05)$ entre M. australis y G. blacodes, pero, significativamente mayores $(\mathrm{P}<0,05)$, respecto a $T$. atun (cuadro 2$)$. La densidad media resultó significativamente mayor $(\mathrm{P}<0,05)$

Cuadro 1. Especies, longitud estándar y peso de peces sin vísceras, comercializados y examinados parasitológicamente en la ciudad de Valdivia, Chile.

Species, standard length, and weight of fish commersialised and examined for parasites in the city of Valdivia, Chile.

\begin{tabular}{lccc}
\hline Especies & $\mathrm{N}^{\circ}$ peces examinados & ${\text { Longitud estándar }(\mathrm{cm})^{\mathrm{a}}}$ & ${\text { Peso total }(\mathrm{g})^{\mathrm{a}}}^{\mathrm{a}}$ \\
\hline Merluccius australis & 104 & $47,1 \pm 7,4$ & $893,1 \pm 357$ \\
"merluza austral" & & $(34-64)$ & $(302-1779)$ \\
\hline Genypterus blacodes & 81 & $66,5 \pm 6,8$ & $1.335 \pm 345$ \\
"congrio dorado" & & $(50-80)$ & $(746-2038)$ \\
\hline Thyrsites atun & 95 & $62,8 \pm 8,6$ & $1.479 \pm 605$ \\
"sierra" & & $(51-96)$ & $(738-3683)$ \\
\hline
\end{tabular}

a media \pm desviación estándar (mínima - máxima). 
Cuadro 2. Peso de los músculos y prevalencia e intensidad media, abundancia media y densidad media de infección en peces comercializados en la ciudad de Valdivia, Chile. Valdivia, Chile.

Weight of muscles, prevalence and mean intensity, mean abundance, and mean density of infection in fish commercialised in the city of

\begin{tabular}{|c|c|c|c|c|c|}
\hline Peces & Peso músculos $(\mathrm{g})^{\mathrm{a}}$ & Prevalencia ${ }^{\mathrm{b}}$ & Intensidad $^{\mathrm{a}}$ & Abundancia $^{\mathrm{a}}$ & Densidad $^{a}$ \\
\hline Merluccius & $406 \pm 162,1$ & 77/104 (74) & $43,6 \pm 111,8$ & 31,9 & 10,1 \\
\hline australis & $(88-994)$ & & $(1-850)$ & & $(250,7)$ \\
\hline Genypterus & $418,6 \pm 122,2$ & $54 / 81(66,7)$ & $19,3 \pm 36$ & 12,8 & 3,3 \\
\hline blacodes & $(178-738)$ & & $(1-211)$ & & (36) \\
\hline Thyrsites & $537 \pm 193$ & $18 / 95(18,9)$ & $17 \pm 44$ & 3,3 & 0,3 \\
\hline atun & $(127-1194)$ & & $(1-149)$ & & $(12,5)$ \\
\hline
\end{tabular}

${ }^{\mathrm{a}}$ media \pm desviación estándar (mínima máxima). ${ }^{\mathrm{b}}$ Peces infectados/examinados (\% prevalencia). Comparaciones entre las tres especies de peces: $\chi 2=$ 68,8 ( $\mathrm{P}<0,05)$; entre M. australis / G. blacodes: $\chi 2=1,2 \mathrm{P}<0,05)$; M. australis / T. atun: $\chi 2=60,4(\mathrm{P}<0,05)$; G. blacodes / T. atun $\chi 2=41,2(\mathrm{P}<0,05)$. Comparaciones de la intensidad media (IM): $\chi 2=16,5(\mathrm{P}<0,05)$, abundancia media (AM): $\chi 2=75,5(\mathrm{P}<0,05)$ y densidad media $(\mathrm{DM}): \chi 2=61,62(\mathrm{P}$ $<0,05)$ entre las tres especies. Comparaciones de IM entre M. australis / G. blacodes $\mathrm{U}=1614,5(\mathrm{P}>0,05)$, M. australis / T. atun $\mathrm{U}=298,5(\mathrm{P}<0,05)$, G. blacodes / T. atun $\mathrm{U}=278,5(\mathrm{P}<0,05)$. Comparaciones de $\mathrm{AM}$ entre M. australis / G. blacodes $\mathrm{U}=3479(\mathrm{P}>0,05)$, M. australis $/$ T. atun $\mathrm{U}=1822,5$ $(\mathrm{P}<0,05)$, G. blacodes / T.atun $\mathrm{U}=1804$ ( $\mathrm{P}<0,05)$. Comparaciones de $\mathrm{DM}$ entre M. australis / G. blacodes $\mathrm{U}=3354,5$ ( $\mathrm{P}<0,05)$, M. australis / T. atun $\mathrm{U}=2357(\mathrm{P}<0,05)$, G. blacodes $/$ T. atun $\mathrm{U}=2562(\mathrm{P}<0,05)$.

en $M$. australis respecto a $G$. blacodes y $T$. atun; en $G$. blacodes la densidad media fue significativamente mayor ( $\mathrm{P}<0,05)$ en relación a $T$. atun (cuadro 2).

El número de taxones parasitarios identificados en cada huésped fluctuó entre 3 y 7 , con cifra mayor en $G$. blacodes (cuadros 2-4). Las metacercarias en M. australis y $G$. blacodes solo fueron observadas mediante la técnica de compresión en placas (cuadros 3-4).

La mayor prevalencia, abundancia media y densidad media de infección correspondieron a Kudoa sp. en $M$. australis. Los demás taxones presentaron prevalencias, abundancias medias y densidades medias de hasta $8,7 \%$, 7,6 y 0,5, respectivamente (cuadro 3). La intensidad media de infección por microsporidios fue 8 veces mayor, respecto a Kudoa sp. (cuadro 3). Pseudoterranova sp. presentó prevalencia, intensidad media y densidad media 10,10 a 14 y 93 a 140 veces mayor que en merluzas y sierras, respectivamente; alcanzando cifras máximas de 35,7 larvas por $100 \mathrm{~g}$ de músculo (cuadro 4).El congrio también presentó larvas de Anisakis sp. tipo I y tipo II con prevalencia, intensidad media, abundancia media y densidad media similares a la infección por Anisakis sp. tipo I de la merluza y la sierra, además de infección por Anisakis tipo II, con descriptores cercanos a los de Anisakis tipo I. En los demás taxones la prevalencia e intensidad, abundancia y densidad media alcanzaron hasta un $21 \%, 6,6,1,4$ y 0,3, respectivamente (cuadro 4). En T. atun las prevalencias de infección por los distintos taxones fluctuaron entre 5,3\% y 8,4\%, sin embargo, la intensidad, abundancia y densidad media de infección por merocercoides de Molicola sp. fueron 20 a 30, 30 y 15 a 30 veces más elevadas que en el caso de las larvas de Pseudoterranova sp. o Anisakis sp. tipo I (cuadro 5).

\section{DISCUSIÓN}

En el presente estudio se registran por primera vez en Chile, Pseudoterranova sp. y Molicola sp. en T. atun y quistes de microsporidios en $M$. australis y G. blacodes . También, se registran por primera vez metacercarias de trematodos en la musculatura de $M$. australis y G. blacodes, asi como, cistacantos de Corynosoma sp. en $G$. blacodes.

De los taxones parasitarios identificados, las larvas de nematodos anisákidos, Pseudoterranova sp. y Anisakis sp. tipos I y II corresponden a aquellas de mayor riesgo de transmisión al hombre (Torres 2012). Las tres especies de peces presentaron infección por nematodos anisákidos y otros parásitos, lo que no se ciñe al artículo 323 del Reglamento Sanitario de Alimentos (RSA 2011) que sostiene que la carne de pescado comercializada para consumo humano debe estar exenta de parásitos y sus quistes; por ello, el pescado fresco comercializado en Chile no debería utilizarse en la preparación de alimentos crudos. El congrio sería la especie de mayor riesgo en la transmisión de anisakidosis al consumidor por presentar la mayor densidad media de infección por Pseudoterranova sp. y por ser la principal causa de anisakidosis humana en Chile (Torres y col 2007). La merluza, especie con elevada cuota de pesca y comercialización nacional y de exportación (Arana 2012), por su mayor densidad media de infección muscular por el total de taxones identificados, puede resultar difícil de comercializar por la presencia de larvas de anisákidos y otros parásitos de efecto repulsivo para el consumidor.

La anisakidosis humana ha sido registrada en distintos países alrededor del mundo donde se consumen alimentos 
Cuadro 3. Prevalencia, intensidad media, abundancia media y densidad media de infección por parásitos en músculos de Merluccius australis $(\mathrm{n}=104)$ comercializados en la ciudad de Valdivia, Chile.

Prevalence, mean intensity, mean abundance, and mean density of infection by parasites in the muscles of Merluccius australis $(\mathrm{n}=104)$ commercialised in the city of Valdivia, Chile.

\begin{tabular}{|c|c|c|c|c|}
\hline Parásitos & Prevalencia $^{\mathrm{a}}$ & Intensidad $^{\mathrm{b}}$ & Abundancia ${ }^{\mathrm{c}}$ & Densidad $^{\mathrm{d}}$ \\
\hline \multicolumn{5}{|l|}{ Trematoda } \\
\hline Digenea gen.sp. (m) & $4(3,8)$ & $\begin{array}{l}2,3 \pm 2,2 \\
(1-6)\end{array}$ & 0.1 & $0,04(1,8)$ \\
\hline \multicolumn{5}{|l|}{ Cestoda } \\
\hline Hepatoxylon trichiuri $(\mathrm{p})$ & $3(2,9)$ & $\begin{array}{l}1 \pm 0 \\
(1-1)\end{array}$ & 0,03 & $0,01(0,2)$ \\
\hline \multicolumn{5}{|l|}{ Nematoda } \\
\hline Pseudoterranova sp.(1) & $5(4,8)$ & $\begin{array}{l}1,4 \pm 0,9 \\
(1-3)\end{array}$ & 0.07 & $0,03(1,1)$ \\
\hline Anisakis sp. tipo I (l) & $9(8,7)$ & $\begin{array}{l}1,1 \pm 0,1 \\
(1-2)\end{array}$ & 0,1 & $0,03(0,5)$ \\
\hline \multicolumn{5}{|l|}{ Myxozoa } \\
\hline Kudoa sp. (q) & $76(73,1)$ & $\begin{array}{l}33,1 \pm 60,6 \\
(1-377)\end{array}$ & 24.2 & $8,1(105)$ \\
\hline Microsporidia gen. sp. (q) & $3(2,9)$ & $\begin{array}{l}263 \pm 446,9 \\
(3-779)\end{array}$ & 7,6 & $0,5(43,5)$ \\
\hline \multicolumn{5}{|c|}{$\begin{array}{l}\mathrm{a}=\text { Prevalencia: peces infectados (porcentaje) }, \mathrm{b}=\text { intensidad media } \pm \text { desviación estándar (mínima-máxima, } \mathrm{c}=\text { abundancia media, } \mathrm{d}=\text { densidad media } \\
\text { por } 100 \mathrm{~g} \text { de músculo (densidad máxima); } \mathrm{m}=\text { metacercarias, } \mathrm{p}=\text { plerocercoides, } 1=\text { larvas del tercer estadio, } \mathrm{q}=\text { quistes con esporas. }\end{array}$} \\
\hline \multicolumn{5}{|c|}{$\begin{array}{l}\text { Cuadro 4. Prevalencia, intensidad media, abundancia media y densidad media de infección por parásitos en músculos de Genypterus } \\
\text { blacodes }(\mathrm{n}=81) \text { comercializados en la ciudad de Valdivia, Chile. } \\
\quad \text { Prevalence, mean intensity, mean abundance, and mean density of infection by parasites in muscles of Genypterus blacodes ( } \mathrm{n}=81 \text { ) }\end{array}$} \\
\hline $\begin{array}{l}\text { Prevalence, mean i } \\
\text { commercialised in the city of Va }\end{array}$ & abundance, and $\mathrm{n}$ & sity of infection & sites in muscles of & rus blacodes (n \\
\hline Parásitos & Prevalencia ${ }^{\mathrm{a}}$ & Intensidad $^{\mathrm{b}}$ & Abundancia ${ }^{\mathrm{c}}$ & Densidad $^{\mathrm{d}}$ \\
\hline \multicolumn{5}{|l|}{ Trematoda } \\
\hline Digenea gen. sp. (m) & $1(1,2)$ & $1(1)$ & 0,01 & $0,002(19)$ \\
\hline \multicolumn{5}{|l|}{ Nematoda } \\
\hline Pseudoterranova sp.(1) & $43(53,1)$ & $\begin{array}{c}20,3 \pm 39,4 \\
(1-207)\end{array}$ & 10,8 & $2,8(35,7)$ \\
\hline Anisakis sp. tipo I (1) & $6(7,4)$ & $\begin{array}{c}1 \pm 0,8 \\
(1-3)\end{array}$ & 0,1 & $0,02(0,4)$ \\
\hline Anisakis sp. tipo II (l) & $4(4,9)$ & $\begin{array}{c}1,8 \pm 1,5 \\
(1-4)\end{array}$ & 0,1 & $0,02(0,8)$ \\
\hline \multicolumn{5}{|l|}{ Acanthocephala } \\
\hline Corynosoma sp. (c) & $1(1,2)$ & $1(1)$ & 0,01 & $0,002(0,1)$ \\
\hline \multicolumn{5}{|l|}{ Myxozoa } \\
\hline Kudoa sp (q). & $17(21)$ & $\begin{array}{c}6,6 \pm 5,9 \\
(1-23)\end{array}$ & 1,4 & $0,3(3,7)$ \\
\hline Microsporidia gen. sp. (q) & $13(16)$ & $\begin{array}{c}3,1 \pm 5,5 \\
(1-21)\end{array}$ & 0,5 & $0,1(4,5)$ \\
\hline
\end{tabular}

$\mathrm{a}=$ Prevalencia: peces infectados (porcentaje), $\mathrm{b}=$ intensidad media \pm desviación estándar (mínima-máxima), $\mathrm{c}=$ abundancia media, $\mathrm{d}=$ densidad media por $100 \mathrm{~g}$ de músculo (densidad máxima); $\mathrm{m}=$ metacercarias, $1=$ larvas del tercer estadio, $\mathrm{c}=$ cistacanto, $\mathrm{q}=$ quistes con esporas. 
Cuadro 5. Prevalencia, intensidad media, abundancia media y densidad media de infección por parásitos en músculos de Thyrsites atun $(\mathrm{n}=95)$ comercializados en la ciudad de Valdivia, Chile.

Prevalence, mean intensity, mean abundance, and mean density of infection by parasites in muscles of Thyrsites atun $(\mathrm{n}=95)$ commercialised in the city of Valdivia, Chile.

\begin{tabular}{|c|c|c|c|c|}
\hline Parásitos & Prevalencia $^{\mathrm{a}}$ & Intensidad $^{\mathrm{b}}$ & Abundanciac $^{c}$ & Densidad $^{\mathrm{d}}$ \\
\hline \multicolumn{5}{|l|}{ Cestoda } \\
\hline Molicola sp. (m) & $8(8,4)$ & $\begin{array}{c}36 \pm 58,4 \\
(1-148)\end{array}$ & 3 & $0,3(12,4)$ \\
\hline \multicolumn{5}{|l|}{ Nematoda } \\
\hline Pseudoterranova sp. (l) & $5(5,3)$ & $\begin{array}{c}1,8 \pm 0,8 \\
(1-3)\end{array}$ & 0.1 & $0,02(0,8)$ \\
\hline Anisakis sp. tipo I (1) & $7(7,4)$ & 1(1) & 0.1 & $0,01(0,3)$ \\
\hline
\end{tabular}

$\mathrm{a}=$ Prevalencia: peces infectados (porcentaje), $\mathrm{b}=$ intensidad media \pm desviación estándar (mínima-máxima), $\mathrm{c}=$ abundancia media, $\mathrm{d}=$ densidad media por $100 \mathrm{~g}$ de músculo (densidad máxima); $\mathrm{m}$ = merocercoides, 1 = larvas del tercer estadio.

a base de pescado crudo (Takahashi y col 1998, Audicana y col 2003, Jimenez-Huyke y col 2005, Shamsi y Butcher 2011), incluido Chile donde la infección ha sido considerada emergente, resultando ser el país sudamericano con mayor número de casos publicados (Torres y col 2007).

La incidencia de anisakidosis humana también se ha incrementado en los últimos años en Estados Unidos y Canadá (Audicana y Kennedy 2008), en especial aquella causada por Pseudoterranova spp., al igual que en Chile (Torres y col 2007). Por el contrario, la anisakidosis por Anisakis spp. es más frecuente en Japón y Europa (Audicana y Kennedy 2008), donde se diagnostican alrededor de 20.000 casos anualmente (Hochberg y Hamer 2010). En España la anisakiosis se considera la infección por nematodos más prevalente, con seroprevalencias de $0,4 \%$ en la región de Galicia y de $15,7 \%$ a $22,1 \%$ en la región sur, atribuyéndose tales diferencias al hábito de consumo de pescado y a la prevalencia de infección en los peces consumidos en ambas regiones (Puente y col 2008). La mayor frecuencia de casos humanos de pseudoterranovosis o anisakiosis en distintas regiones geográficas podría estar relacionada con variaciones en la distribución geográfica de las especies de Anisakis y Pseudoterranova (Mattiucci y Nascetti 2008). En Chile, la fuente de infección habitualmente se encuentra asociada al consumo de cebiche y pescado frito (Torres y col 2007), registrándose también algunos casos asociados al consumo de pescado ahumado (Mercado y col 1997) o sushi (Mercado y col 2001). En el 80\% de los casos conocidos en Chile, el paciente ha eliminado las larvas por la boca, en el resto de los casos las larvas han sido extraídas por gastroscopia o han sido eliminadas con las heces (Torres y col 2007).

Las larvas de anisákidos en los peces comercializados también tiene un impacto económico sobre la industria por la pérdida que ocasiona su detección y extracción de los músculos del bacalao (Gadus morhua) y otros peces demersales como ha sido demostrado en la costa atlántica de Canadá, calculándose una pérdida de 27 a 50 M de dólares anualmente (McClelland 2002).

La diferenciación de las L3 de las especies de Pseudoterranova y Anisakis es posible mediante la aplicación de pruebas moleculares, las características morfológicos utilizadas en el presente trabajo (Petter y Maillard 1988) son similares para las 8 especies conocidas, $P$. decipiens (sensu stricto), P. azarasi, P. bulbosa, P krabei, P. ceticola, $P$. cattani, $P$. kogiae y $P$. decipiens E (Mattiucci y Nascetti 2008). Las características morfológicas de las L3 de Anisakis tipo I (Berland (1961) son similares para A. simplex (sensu stricto), A. pegreffi, Anisakis simplex C, A. typica, A. nascetti y A. ziphidarum, mientras que aquellas de Anisakis tipo II (Berland 1961) pueden corresponder a A. physeteris, A. brevispiculata y A. paggiae (Mattiucci y Nascetti 2008). En Chile, se han identificado L3 de P. cattani, A. pegreffi y A. simplex $\mathrm{C}$ en peces, mediante técnicas moleculares (Mattiucci y Nascetti 2008).

Las diferencias respecto a los descriptores de infección entre los distintos huéspedes, podría estar asociado a las diferencias de ambientes que ocupan, G. blacodes (bentónico), M. australis (bento-demersal) y T. atun (pelágico) lo que determina su dieta y por ende los tipos de huéspedes intermediarios/paratenicos que transmiten la infección. En el hemisferio norte, las L3 de Pseudoterranova decipiens (sensu lato) se presentan en diversos invertebrados que sirven de alimento a los peces, tales como crustáceos copépodos, misidáceos, anfípodos, isópodos, decápodos y cumáceos, así como, poliquetos y gasterópodos (Anderson 2000). Las L3 de Anisakis simplex (s. l.) han sido identificadas en crustáceos eufaúsidos y en cefalópodos (Anderson 2000). En Chile, la dieta de $G$. blacodes está principalmente constituida por el langostino colorado (Bahamonde y Zavala 1981) a diferencia de 
M. australis que consume principalmente crustáceos eufaúsidos y copépodos y T. atun que consume principalmente eufaúsidos, así como peces teleósteos (Bahamonde 1951). El papel de dichos invertebrados en la transmisión de anisákidos a los peces se desconoce en Chile.

Solo en dos de los estudios previos sobre las especies de peces examinados (Muñoz y Olmos 2008) se especificó el procedimiento de búsqueda (técnica de candling) de los parásitos en los músculos, encontrándose uno de cinco ejemplares de G. blacodes infectados con Anisakis sp. (Torres y col 1983) y prevalencias de 3\% e intensidad y abundancia media de 1,5 y $<0,1$ de infección por P. decipiens (sensu lato), respectivamente en $M$. australis (González y Carvajal 1994). Entre los helmintos también se menciona la presencia de $P$. decipiens en músculos de G. blacodes y Lacistorhynchus sp., Grillotia heptanchi, Hepatoxylon trichiuri y Kudoa sp. en M. australis (González y Carvajal 1994, Muñoz y Olmos 2008).

En Chile, el pescado fresco es refrigerado (entre $0 \mathrm{y}$ $4{ }^{\circ} \mathrm{C}$ ) o es tratado con un golpe de frío alcanzando hasta $-3{ }^{\circ} \mathrm{C}$ en la superficie del producto (SERNAPESCA 2011), sin embargo, dichas temperaturas permiten que las larvas de anisákidos permanezcan viables. Las larvas de anisákidos mueren en la carne mediante cocción sobre $65{ }^{\circ} \mathrm{C}$ por 10 min como mínimo y en microondas a $77^{\circ} \mathrm{C}$ (Adams y col 1999) o por congelamiento a $-20{ }^{\circ} \mathrm{C}$ por $7 \mathrm{~d}$ o $-35^{\circ} \mathrm{C}$ o menos hasta congelar, almacenando a -35 ${ }^{\circ} \mathrm{C}$ o menos por $15 \mathrm{~h} \mathrm{o} \mathrm{a}-20^{\circ} \mathrm{C}$ o menos por $24 \mathrm{~h}$, medidas adecuadas para peces de hasta $15,2 \mathrm{~cm}$ de grosor (FDA 2011). La Comunidad Europea recomienda $-20^{\circ} \mathrm{C}$, aplicada en todas las partes del producto, por no menos de 24 h (Audicana y Kennedy 2008). Sin embargo, las aplicaciones reseñadas pueden variar según el huésped, el parásito y las porciones del pescado en procesamiento (Adams y col 2005). Lo importante en la aplicación térmica es que alcance todas las partes del producto y para ello es posible que en algunos casos se requiera más de $24 \mathrm{~h}$ (Wharton y Aalders 2002). Aunque, el tratamiento térmico del pescado previene la transmisión de larvas de anisákidos, los antígenos del parásito pueden resultar termoestables, lo que no evita las reacciones alérgicas (Audicana y Kennedy 2008, Ventura y col 2008). RodriguezMahillo y col (2010) observaron que los alergenos de $A$. simplex se preservan en los peces por 11 min a $-20{ }^{\circ} \mathrm{C}$ y también en las merluzas después de cocinar. Las temperaturas elevadas provocan daño en la cutícula de las larvas resultando susceptibles a la acción enzimática del estómago, causando su muerte y liberación de antígenos somáticos (Adams y col 1999, Tejada y col 2006). El uso de presión hidrostática, como método alternativo, ha sido eficiente en el tratamiento de la carne para matar las larvas de A. simplex (Molina-García y Sanz 2002, Brutti y col 2010), aunque, su aplicación a filetes de salmón afecta el color de la carne (Dong y col 2003). También, el tratamiento con ácido acético de $10 \%$ y lavado por una hora puede garantizar la destrucción de las larvas de Ani- sakis spp. en Engraulis encrasicholus, resultando más económico, sin afectar la apariencia, color y textura de la carne (Sanchez-Monsalvez y col 2005).

De los demás taxones identificados en los peces examinados, ninguno ha sido comprobado como causante de zoonosis, aunque en algunos casos otras especies pertenecientes a los géneros u otros taxones identificados en el presente estudio también han sido relacionados con zoonosis pero de manera accidental. Así por ejemplo, solo se ha registrado un caso accidental de infección por el acantocéfalo Corynosoma strumosum en un esquimal de Alaska (Coombs y Crompton 1991) y un caso en un niño que eliminó espontáneamente con sus heces un plerocercoide de una especie no identificada de Нераtoxylon, ingerida al consumir pescado crudo (Deardorff y col 1984). Asimismo, los productos somáticos de merocercoides de Gymnorhynchus gigas (Trypanorhyncha) pueden causar reacciones alérgicas en un modelo murino (Rodero y Cuéllar 2000) y los estudios experimentales en ratones sugieren que los extractos crudos de merocercoides de Molicola horridus contienen moléculas alergénicas que constituyen riesgo para los consumidores de pescado (Gómez-Morales y col 2008, Pelayo y col 2009). Martínez de Velasco y col (2008), en pacientes con síntomas gastrointestinales o alérgicos, observaron reacción cutánea a extractos de Kudoa sp., después de consumir pescado. En Japón, se identificó K. septempunctata como responsable de repetidos brotes de gastroenteritis ocurridos desde 2003 y asociados al consumo del lenguado P. olivaceus (Kawai y col 2012). En el presente estudio, la presencia de Kudoa sp. en un elevado porcentaje de merluzas así como en un porcentaje menor de congrios hace necesario estudiar el posible efecto de estos parásitos en modelos experimentales, de manera similar a K. septempunctata (Kawai y col 2012), con el fin de evaluar su potencial riesgo para la salud.

Algunos autores plantean que el consumo de pescado crudo podría favorecer la transmisión de microsporidiosis humana (Slifko y col 2000). De las 15 especies de microsporidios identificadas en el hombre (Didier 2005, Choudhary y col 2011, Meissner y col 2012), solo Pleistophora ronneafiei pertenece a un género que incluye 26 especies (Lom 2002) descritas en músculos de peces. Sin embargo, actualmente se desconoce la fuente de infección humana por $P$. ronneafiei, sospechándose que corresponda muy probablemente a una especie de pez (Cali y Takvorian 2003, Liu 2011). Finalmente, el escaso número de metacercarias encontradas en los músculos de las merluzas y congrios podrían corresponder a estados que completan su desarrollo en peces, aves o mamíferos predadores y su papel como zoonosis requiere de trabajos experimentales. En Chile, los estudios de metacercarias en músculos de peces marinos o de estuarios son muy escasos registrándose solo metacercarias de Manteria sp., en el chancharro, Caprodon longimanus, y de Ascocotyle (Phagicola) longa en la lisa Mugil cephalus (Muñoz y 
Olmos 2008), esta última especie es causa de una zoonosis emergente en el hombre y desarrolla su estado adulto en mamíferos y aves piscívoras presentando sus estados larvarios en el molusco Heleobia australis y en peces (Mugil spp.) (Portes-Santos y col 2013). En Chile, la infección por Ascotyle (Phagicola) sp. ha sido registrada en gatos y perros de la ciudad de Valdivia (Torres y col 1972,1974), en un 91,4\% de ejemplares examinados de M. cephalus de Concepción (Fernández 1987) y en perros y casos humanos en Brasil (Chieffi y col 1990, 1992).

Los resultados confirman en tres especies de peces que son de alto consumo en el sur de Chile, la existencia de distintos parásitos eucarióticos, algunos causantes de zoonosis que representan una fuente de infección para los consumidores de pescado crudo. En Japón, el personal que procesa y prepara el pescado debe cumplir con estrictos estándares para recibir una licencia gubernamental y desempeñarse laboralmente (Kure y Yokoi 1989). En España, se exige congelar el pescado, previo a la preparación de platos crudos (BOE, 2006), derivado de la alta incidencia de anisakidosis (Audicana y Kennedy 2008). En Chile, debido al incremento del consumo de pescado crudo y las características emergentes de la anisakidosis, debería exigirse la congelación del pescado previo a la preparación de alimentos crudos. Asimismo, debería capacitarse y certificar el desempeño de las personas que procesan, limpian y preparan el pescado crudo, con conocimiento para separar eventuales larvas de los parásitos durante su manipulación. Con lo anterior, debe reforzarse la educación sanitaria de la población para crear conciencia sobre el adecuado tratamiento térmico del pescado y así lograr su inocuidad, evitando riesgos para su salud.

\section{REFERENCIAS}

Adams AM, KD Murrell, JH Cross. 1997. Parasites of fish and risk to public health. Rev Sci Tech Off Int Epiz, 10, 652660.

Adams AM, KS Miller, MM Wekell, FM Dong. 1999. Survival of Anisakis simplex in microwave-processed arrowtooth flounder (Atheresthes stomias). J Food Prot 62, 403-409.

Adams AM, MN Ton, M Marleen, A Wekell, P Mackenzie, FM Dong. 2005. Survival of Anisakis simplex in arrowtooth flounder (Atheresthes stomia) during frozen storage. $J$ Food Prot 68, 1441-1446.

Anderson RC. 2000. Nematode parasites of vertebrates. CABI Publishing, Oxon, UK.

Arana P. 2012. Recursos pesqueros del mar de Chile. Ediciones Universitarias de Valparaíso. Pontificia Universidad Católica de Valparaíso, Valparaíso, Chile.

Audicana MT, MD del Pozo, R Iglesias, FM Ubeira. 2003. Anisakis simplex and Pseudoterranova decipiens. Food Sci Tech 125, 613-636

Audicana MT, MW Kennedy. 2008. Anisakis simplex: from obscure infections worm to inducer of immune hypersensitivity. Clin Microbiol Rev 21, 360-379.

Bahamonde N. 1951. Alimentación de la sierra (Thyrsites atun)
(Euphrasen 1791). Invest Zool Chil 1, 8-10.

Bahamonde N, P Zabala. 1981. Contenidos gástricos de Genypterus maculatus (Tschudi) y Genypterus blacodes (Schneider) capturados en Chile entre $31^{\circ}$ y $37^{\circ} \mathrm{S}$ (Teleostomi, Ophidiidae). Bol Mus Nac Hist Nat Chile 3, 53-59.

Berland B. 1961. Nematodes from some Norwegian marine fishes. Sarsia 2, 1-50.

BOE, Boletín Oficial de España, España. 2006. Real decreto 1420/2006, N 302, Pp 445-447.

Broglia A, C Kapel. 2011. Changing dietary habits in a changing world: emerging drives for the transmission of foodborne parasitic zoonoses. Vet Parasitol 182, 2-13.

Brutti A, P Rovere, S Cavallero, SD Amelio, P Danesi, S Arcangeli. 2010. Inactivation of Anisakis simplex larvae in raw fish using high hydrostatic pressure treatments. Food Control 21, 331-333.

Bush AO, KD Lafferty, JM Lotz, AW Shostak. 1997. Parasitology meets ecology on its own terms: Margolis et al. revisited. J Parasitol 83, 575-583.

Cali A, PM Takvorian. 2003. Ultrastructure and development of Pleistophora ronneafiei n.sp., a microsporidium (Protista) in the skeletal muscle of an immune-compromised individual. J Eukaryot Microbiol 50, 77-85.

Ceballos-Mandiola G, A Valero, R Polo-Vico, M Tejada, N Abattouy, H Karl, C de las Heras, J Martín-Sanchez. 2010. Genetic variability of Anisakis simplex s.s. parasitizing European hake (Merluccius merluccius) in the little sole Bankarea in the Northeast Atlantic. Parasitol Res 107, 1399-1404.

Chieffi PP, OH Leite, RMD Souza-Días, DMA Vieira Torres, ACS Mangini. 1990. Human parasitism by Phagicola sp. (Trematoda, Heterophyidae) in Cananéia, Sao Paulo State, Brazil. Rev Inst Med Trop Sao Paulo 32, 285-288.

Chieffi PP, MCO Gorla, DMAG Vieira-Torres, RMD Souza-Dias, ACS Magnini, AV Monteiro, E Woiciechovski. 1992. Human infection by Phagicola sp. (Trematoda, Heterophyidae) in the municipality of Registro, Sao Paulo State, Brazil. J Trop Med Hyg 95, 346-348.

Choudhary MM, MG Metcalfe, K Arrambide, C Bern, GS Visvesvara, NJ Pieniazek, RD Bandea, M DeLeon-Carnes, P Adem, MM Choudhary, SR Zaki, MU Saeed. 2011. Tubulinosema sp. microsporidian myositis in immunosuppressed patient. Emerg Infect Dis 17, 1727-1730.

Coombs I, DWT Crompton. 1991. A guide to human helminthes. Taylor and Francis Press, London, UK.

Deardorff TL, RB Raybourne, TE Mattis. 1984. Infections with Trypanorhyncha plerocerci (Cestoda) in Hawaiian fishes of commercial importance. Q Univ Hawai Sea Grant Coll Prog 6, 1-6.

Didier ES. 2005. Microsporidiosis: an emerging and opportunistic infection in humans and animals. Act Trop 94, 61-76

Dong FM, AR Cook, RP Herwing. 2003. High hydrostatic pressure treatment of finfish to inactivate Anisakis simplex. $J$ Food Prot 66, 1924-1926.

FDA, Food and Drug Administration, USA. 2009. Method for determination of parasites in fin fish. Macroanalytical procedures manual V-7. Seafood, Pp 1-5.

FDA, Food and Drug Administration, USA. 2011. Fish and fishery products hazards and controls guidance. Chapter 5. Parasites, Pp 91-98.

Fernández J. 1987. Los parásitos de la lisa Mugil cephalus L., 
en Chile: sistemática y aspectos poblacionales (Perciformes: Mugilidae). Gayana 51, 3-58.

Gómez-Morales MA, A Ludovsi, E Giuffia, MT Manfredi, G Piccolo, E Pozio. 2008. Allergenic activity of Molicola horridus (Cestoda, Trypanorhyncha) a cosmopolitan fish parasite, in a mouse model. Vet Parasitol 157, 314-320.

González L, Carvajal J. 1994. Estudio parasitológico de Merluccius australis (Hutton, 1872) del mar interior de Aysén. Invest Pesq 38, 75-85.

Hochberg NS, DH Hamer. 2010. Anisakidosis: perils of the deep. Clin Infect Dis 51, 806-812.

Jimenez-Huyke CE, S Rivera, F Rodriquez, M Marcial. 2005. Anisakiasis associated to raw fish ingestion. Am J Gastroenterol 100, S191.

Jofré L, P Neira, I Noemí, L José, C Cerva. 2008. Pseudoterranovosis y sushi. Rev Chil Infect 25, 200-206.

Kawai T, T Sekizuka, Y Yahata, M Kuroda, Y Kumeda, Y Lijima, Y Kamota, Y Sugita-Konishi, T Ohnishi. 2012. Identification of Kudoa septempunctata as the causative agent of novel food poisoning outbreaks in Japan by consumption of Paralichthys olivaceus in raw fish. Clin Infect Dis 54, 1046-1052.

Kleter GA, A Prandini, L Filippi, HJP Marvin. 2009. Identification of potentially emerging food safety issues by analysis of reports published by the European Community's Rapid Alert System for Food and Feed (RASFF) during a fouryear period. Food Chem Toxicol 47, 932-950.

Kure K, M Yokoi. 1989. Safe sushi. N Engl J Med 321, 900901.

Lin AH, E Flowaag, T Van Do, SGO Johansson, A Levsen, K Vaoli. 2012. IgE sensitization to the fish parasite Anisakis simplex in a Norwegian population: a pilot study. Scand $J$ Inmunol 75, 431-435.

Liu D. 2011. Pleistophora and Trachipleistophora. In: Liu D (ed). Molecular detection of human fungal pathogens. CRS Press, Boca Raton, USA, Pp 843-848.

Llarena-Reino M, AF González, C Vello, L Outeriño, S Pascual. 2012. The accuracy of visual inspection for preventing risk of Anisakis sp. infection in unprocessed fish. Food Control 23, 54-58.

Lom J. 2002. A catalogue of described genera and species of microsporidians parasitic in fish. Syst Parasitol 53, 81-99

Martínez de Velasco G, M Rodero, C Cuéllar, T Chivato, JM Mateos, R Laguna. 2008. Skin prick test of Kudoa sp. antigens in patients with gastrointestinal and/or allergic symptoms related to fish ingestion. Parasitol Res 103, 713-715.

Mattiucci S, G Nascetti. 2008. Advances and trends in the molecular systematics of anisakid nematodes with implications for their evolutionary ecology and host-parasite coevolutionary processes. Adv Parasitol 66, 47-148.

McClelland G. 2002. The trouble with sealworms (Pseudoterranova decipiens species complex, Nematoda): a review. Parasitology 124, 183-203

Meissner EG, JE Bennett, I Qvarnstrom, A da Silva, EY Chu, M Tsokos, J Gea-Banacloche. 2012. Disseminates microsporidiosis in an immunosuppressed patient. Emerg Infect Dis 18, 1155-1158.

Mercado R, P Torres, J Maira. 1997. Human case of gastric infection by a fourth larval stage of Pseudoterranova decipiens (Nematoda, Anisakidae). Rev Saúde Públ 31, 178-181.
Mercado R, P Torres, V Muñoz, W Apt. 2001. Human infection by Pseudoterranova decipiens (Nematoda, Anisakidae) in Chile: report of seven cases. Mem Inst Oswaldo Cruz 96, 653-655.

Molina-García AD, PD Sanz. 2002. Anisakis simplex larva killed by high-hydrostatic-pressure processing. J Food Prot 65, 383-388.

Muñoz G, V Olmos. 2008. Revisión bibliográfica de especies endoparásitas y hospedadores de sistemas acuáticos de Chile. Rev Biol Mar Oceanogr 43, 173-245.

Palm HW. 2004. The Trypanorhyncha Diesing, 1863. PKSPLIPB Press, Bogor, Indonesia.

Pelayo V, P García-Hernández, P Puente, M Rodero, C Cuellar. 2009. Seroprevalence of Anti- Gymnorhynchus gigas (Trypanorhyncha, Gymnorhynchidae) antibodies in a Spanish population. J Parasitol 95, 778-780.

Petter AJ, C Maillard. 1988. Larves d'ascarides parasites de poissons en Méditerranée occidentale. Bull Mus Natn Hist Nat Paris 10, 347-369.

Portes-Santos C, Correa-Lopes K, Silva-Costa V, EG Nunes dos Santos. 2013. Fish-borne trematodosis: potential risk of infection by Ascocotyle (Phagicola) longa (Heterophyidae). Vet Parasitol 193, 302-306.

Puente P, AM Anadón, M Rodero, F Romarís, FM Ubeira, C Cuellar. 2008. Anisakis simplex: the high prevalence in Madrid (Spain) and its relation with fish consumption. Exp Parasitol 118, 271-274

Robinson ES. 1959. Some new cestodes from New Zeland marine fishes. Trans $R$ Soc $N$ Z 86, 381-392.

Rodero M, C Cuéllar. 2000. IgE antibody to parasite induced in mice. Allergy 55, 784-785.

Rodriguez-Mahillo Al, I Rodriquez-Mahillo, M González-Muñoz, C de las Heras, M Tejada, I Moneo. 2010. Quantification of Anisakis simplex allergens in fresh, long-term frozen, and cooked fish muscle. Foodborne Pathog Dis 7, 967-973.

RSA, Reglamento Sanitario de los Alimentos, Chile. 2011. Título XII. De los pescados. Artículo 23.

Sachs L. 1968. Estadística Aplicada. Editorial Labor, Madrid, España.

Sagua H, I Neira, J Araya, J González. 2001. Nuevos casos de infección humana por Diphyllobothrium pacificum (Nybelin, 1931) Margolis, 1956 en Chile y su probable relación con el fenómeno de El Niño, 1975-2000. Bol Chil Parasitol 56, 22-25.

Sánchez-Monsalvez I, C de Armas-Serra, J Martínez, M Dorado, A Sánchez, F Rodríquez-Caabeiro. 2005. A new procedure for marinating fresh anchovies and ensuring the rapid destruction of Anisakis larvae. J Food Prot 68, 1066-1072.

SERNAPESCA, Servicio Nacional de Pesca, Chile. 2011. Requisitos sanitarios y planes de muestreo para la certificación sanitaria de productos pesqueros de exportación. Sección 2. Departamento de Sanidad Pesquera, Pp 1-101.

SERNAPESCA, Servicio Nacional de Pesca, Chile. 2012. Métodos de análisis físico-químicos para productos pesqueros de exportación. Programa de Laboratorios, norma técnica. Sección 2. Departamento de Sanidad Pesquera. Pp 1-20.

Shamsi S, A Butcher. 2011. First report of human anisakidosis in Australia. MJA 194, 199-200.

Slifko TR, HV Smaith, JB Rose. 2000. Emerging parasite zoo- 
noses associated with water and food. Int J Parasitol 30, 1379-1393.

Smith JW, R Wootten. 1975. Experimental studies on the migration of Anisakis sp. larvae (Nematoda: Ascaridia) into the flesh of herring, Clupea harengus L. Int J Parasitol 5, 133-136.

Takahashi S, H Ishikura, K Kibuchi. 1998. Anisakidosis: global point of view. In Ishikura H, M Aikawa, H Itakura, K Kikuchi (eds). Host response to international parasitic zoonoses. Springer-Verlag, Tokio, Japan, Pp 109-120.

Tejada M, MT Solis, A Navas, A Mendizábal. 2006. Scanning electron microscopy of Anisakis larvae following different treatments. J Food Prot 69, 1379-1387.

Torres P, A Hott, H Boehmwald. 1972. Protozoos, helmintos y artrópodos en gatos de la ciudad de Valdivia y su importancia para el hombre. Arch Med Vet 4, 20-29.

Torres P, M Ramos, L Carrasco, M Neumann, R Franjola, N Navarrete, L Figueroa. 1974. Protozoos, helmintos y artrópodos parásitos del perro doméstico en la ciudad de Valdivia, Chile. Arch Med Vet 29, 18-23.

Torres P, E Hernández, I Sandoval. 1983. Anisakiasis and phocanemiasis in marine fishes from de south of Chile. Int $J$ Zoon 10, 146-150.

Torres P, R Moya, J Lamilla. 2000. Nematodos anisákidos de interés en salud pública en peces comercializados en Valdivia, Chile. Arch Med Vet 32, 107-113.

Torres P, MI Jercic, JC Weitz, EK Dobrew, R Mercado. 2007. Human pseudoterranovosis, an emerging infection in Chile. J Parasitol 93, 440-443.

Torres P, JC Quintanilla, M Rozas, P Miranda, M Ibarra, MF San Martín, B Raddatz, M Wolter, A Villegas, C Canobra, M Hausdorf, R Silva. 2010. Endohelminth parasites from salmonids in intensive culture from southern Chile. J Parasitol 96, 669-670.

Torres P, S Puga. 2011. Comparative efficacy of candling and glass plate compression for detection of diphyllobothriosis in rainbow trout (Oncorhynchus mykiss) musculature. Rev Sci Tech Off Int Epiz 30, 831-837.

Torres P. 2012. Parásitos eucarióticos de peces y su importancia en la salud. En: Canals M, PE Cattan (eds). Zoología Médica III. Vertebrados. Editorial Universitaria, Santiago, Chile, Pp 213-265.

Ventura MT, RA Tummolo, E Di Leo, M D`Ersasmo, A Arsieni. 2008. Immediate and cell-mediated reactions in parasitic infections by Anisakis simplex. J Investig Allergol Clin Immunol 18, 253-259.

Wharton DA, O Aalders. 2002. The response of Anisakis larvae to freezing. J Helminthol 76, 363-368. 